\title{
Ultrasound and Microbubble Gene Delivery for Targeting Altered Placental MicroRNAs in Preeclampsia
}

\author{
Sonya Frazier \\ Institute of Cardiovascular and \\ Medical Sciences \\ University of Glasgow \\ Glasgow, UK \\ s.frazier.1@ research.gla.ac.uk
}

\author{
Angela Bradshaw \\ Institute of Cardiovascular and \\ Medical Sciences \\ University of Glasgow \\ Glasgow, UK \\ Angela.Bradshaw@glasgow.ac.uk
}

\author{
Hannah Morgan \\ Division of Child Health, Obstetrics \\ and Gynaecology \\ University of Nottingham \\ Nottingham, UK \\ Hannah.Morgan@nottingham.ac.uk
}

\author{
Helen Mulvana \\ Biomedical Engineering \\ University of Strathclyde \\ Glasgow, UK \\ Helen.Mulvana@strath.ac.uk
}

\author{
Martin McBride \\ Institute of Cardiovascular and \\ Medical Sciences \\ University of Glasgow \\ Glasgow, UK \\ Martin.McBride@glasgow.ac.uk \\ Delyth Graham \\ Institute of Cardiovascular and \\ Medical Sciences \\ University of Glasgow \\ Glasgow, UK \\ Delyth.Graham@glasgow.ac.uk
}

\begin{abstract}
Ultrasound (US) and microbubble (MB) gene delivery has attracted growing interest as a clinically applicable gene therapy (GT). Though preclinical studies have investigated the system in various tissues, there is limited research in targeting the placenta. This is a potential therapeutic strategy for preeclampsia (PE), which has an underlying genetic basis and ineffective management strategies. Differentially expressed placental microRNAs (miRNAs) in PE may represent suitable targets for GT. Microbubbles (SonoVue) and plasmid (pGL3 or pGL4.13) were administered systemically to CD1 mice, followed by exposure of the heart to US (B-mode, H14, 1.8 M.I., $1 \mathrm{~cm}$ focal depth, 2 minutes), using Siemens Acuson Sequoia-512 system and $15 \mathrm{LB}$ probe. Luciferase assays were performed to evaluate gene transfection. Significantly differentially expressed placental miRNAs in PE patients were identified as candidate miRNAs based on detection by $\geq 3$ screening studies. Expression of candidate miRNAs was measured by qRT-PCR in PE rat model placentas. In trial 1, low levels of luciferase activity were detected in the heart of treatment mouse 1, 2 and 3. In trial 2, luciferase activity was evident in the atria of treatment mouse 2 . In trial 3, higher luciferase activity was detected in the ventricles of the treatment mouse and activity was also detected in the atria. The literature review identified eight candidate miRNAs. MiR-223 (1.46-fold increase) and miR-181a (0.81-fold decrease) were significantly differentially expressed in $\mathrm{PE}$ rat model placentas. MiR-223 and miR-181a may represent targets for US and MB gene delivery. Future studies will apply the US and MB gene delivery protocol for translation to targeting the placenta in our PE rodent model.
\end{abstract}

Keywords-Ultrasound, Microbubbles, Gene Therapy, Preeclampsia, Placenta, MicroRNAs

\section{INTRODUCTION}

Substantial progress has been made in the field of gene therapy (GT), with six therapies approved since 2012 [1] and the number of clinical trials continuing to rise [2]. GT is defined as the transfer of genetic material into cell nuclei as a treatment for diseases with a genetic component. To target desired cells and achieve therapeutically relevant gene transfer for a sufficient time period, gene delivery systems are employed, typically: viral or non-viral [3]. Viral-based systems generally lead to higher transduction efficiencies but carry serious safety risks [3]. Non-viral systems are considered safer but suffer lower transfection efficiencies; thus, physical and/or chemical methods are often used to enhance transfection levels [3]. Despite technological advancements, the majority of trials remain in phase I and I/II [2]. Hence, preclinical proof-of-concept studies are crucial to the development of safe and effective gene delivery systems.

Ultrasound (US) and microbubble (MB) gene delivery has attracted growing interest as a clinically applicable GT. In conjunction with plasmid DNA or other nucleic acid complexes, US and MB gene delivery is a non-viral physical method for enhancing gene transfection. In preclinical studies, the delivery system has been used to target various tissues and therapeutically relevant gene transfer levels have been reported in numerous diseases $[4,5]$. However, there is limited research in targeting the placenta.

Preeclampsia (PE) is a pregnancy disorder, characterized by new hypertension and significant proteinuria at or after 20 weeks of pregnancy [6]. It is a leading cause of maternal and perinatal morbidity and mortality [6]. Delivery resolves PE's complications; however, preterm deliveries increase the risk of foetal morbidity and mortality [6]. There are treatments that target maternal complications to delay delivery although these carry safety and efficacy issues [7-9]. Hence, there is a need for novel treatment strategies that can prevent PE.

$\mathrm{PE}$ is now recognised as a complex genetic disorder, with numerous candidate genes identified [10] and growing evidence of altered placental microRNA (miRNA) expression profiles in PE patients [11]. MiRNAs are short non-coding RNAs involved in post-transcriptional gene regulation. Invitro and in-vivo studies have demonstrated their role in placental development and function as well in the pathology of PE arising from their aberrant expression [12, 13].

The aims of this study were 1) to develop a protocol for US and MB gene delivery with potential applicability to the placenta by conducting proof-of-concept trials to demonstrate site-specific gene transfection in the hearts of CD1 mice and 2) to identify differentially expressed placental miRNAs in 3rd trimester PE patients, through a literature review, and compare their expression with that of PE rodent model recently published by our group [14]. 


\section{METHODS}

\section{A. Plasmid DNA}

The pGL3-Control Vector (Promega, Southampton, UK), a plasmid expressing luc+, a modified firefly luciferase gene, was transformed into MAX Efficiency ${ }^{\mathrm{TM}} \mathrm{DH} 5 \alpha^{\mathrm{TM}}$ Competent Cells (ThermoFisher, Paisley, UK). pGL3 was isolated and purified using Invitrogen ${ }^{\mathrm{TM}}$ PureLink ${ }^{\mathrm{TM}}$ HiPure Expi Plasmid Megaprep Kit, according to the Revision A.0 User Guide (ThermoFisher, Vilnus, Lithuania). From a glyceral stock, the pGL4.13[luc2/SV40] Vector, a plasmid expressing luc2, a synthetically engineered luciferase gene, was isolated and purified using Invitrogen ${ }^{\mathrm{TM}}$ PureLink ${ }^{\mathrm{TM}}$ HiPure Expi Plasmid Megaprep Kit, according to the Revision A.0 User Guide (ThermoFisher, Vilnus, Lithuania). Plasmid DNA concentration was measured using NanoDrop 1000 Spectrophotometer (ThermoFisher, Paisley, UK) and adjusted with $1 \mathrm{X}$ Tris-EDTA (TE) buffer to obtain $4 \mu \mathrm{g} / \mu \mathrm{L}$.

\section{B. Plasmid DNA and Microbubble Suspension}

SonoVue (Bracco International B.V., Amsterdam, Netherlands) was prepared according to the manufacturers instruction at double concentration, by discarding half the volume $(2.5 \mathrm{ml})$ of sodium chloride $(0.9 \%) .150 \mu \mathrm{L}$ of $\mathrm{MBs}$ and $50 \mu \mathrm{L}$ of plasmid DNA were pipetted into a microcentrifuge tube, making up a $200 \mu \mathrm{L}$ injectable suspension per animal.

\section{In-vivo Transfection}

All animal work was carried out in accordance with the Animals (Scientific Procedures) Act 1986. Female, 6-8 week old, CD-1 mice $(25 \mathrm{~g}-35 \mathrm{~g})$ were anaesthetised with $5 \%$ isofluorane and maintained on $1.5 \%$ isofluorane on a homeothermic mat. Mice were placed in a supine position, and, over an area of $1.5 \mathrm{~cm}^{2}$ above the ribcage, hair was removed by shaving and depilatory cream, and US gel was applied. For all US scans, Acuson Sequoia-512 ultrasound system (Siemens pls, Surrey, UK) and 15L8 linear array transducer probe with a frequency range of 7-15 MHz was used. B-mode output was used for organ location. The transducer was placed on the shaved area overlying the heart to obtain a parasternal long axis view (14 MHz, 0.6 mechanical index (MI), and $10 \mathrm{~mm}$ focal depth). Once the organ was located and appropriate view obtained, the probe was held in a fixed position and set to harmonic imaging (H14 $\mathrm{MHz}, 1.8 \mathrm{MI}$, and $10 \mathrm{~mm}$ focal depth). For treatment mice, the plasmid DNA and MB suspension was gently stirred with a $25 \mathrm{G}$ needle (Terumo, Leuven, Belgium) and drawn into a syringe (Table 1). The suspension was injected slowly into the tail vein over a 60 second period. This was immediately followed by an additional 2 minutes of US application. The probe was then removed and the mouse was recovered. Negative control mice underwent the same procedure, receiving 1X phosphate buffered saline (PBS) / $\mathrm{MB}$ suspension in place of plasmid DNA (Table 1). Positive control mice were anaesthetised with $5 \%$ isofluorane and maintained on $1.5 \%$ isofluorane on a homeothermic mat. An area of $1 \mathrm{~cm}^{2}$ over the left gracilis muscle was shaved and plasmid DNA / MB suspension was administered into the gracilis muscle by multiple intramuscular injections (Table 1). The mouse was then recovered. All mice were sacrificed after three days, and the hearts and left gracilis muscles were dissected and snap frozen in liquid nitrogen.
TABLE I. EXPERIMENTAL DESIGN OF IN-VIVO TRIALS

\begin{tabular}{|l|l|c|c|}
\hline \multirow{2}{*}{$\begin{array}{c}\text { Trial } \\
\text { plasmid) }\end{array}$} & \multicolumn{3}{|c|}{ Experimental Design } \\
\cline { 2 - 4 } 1 (pGL3) & Treatment (3) & $\begin{array}{c}\text { Plasmid } \\
\text { volume }(\boldsymbol{\mu L})\end{array}$ & $\begin{array}{c}\text { Microbubble } \\
\text { volume }(\boldsymbol{\mu L})\end{array}$ \\
\hline 1 (pGL3) & Negative Control (1) & $200 \mu \mathrm{L} \mathrm{PBS}$ & 150 \\
\hline 1 (pGL3) & Positive Control (1) & 50 & 0 \\
\hline 2 (pGL3) & Treatment (3) & 50 & 150 \\
\hline 2 (pGL3) & Negative Control (1) & $160 \mu \mathrm{L} \mathrm{PBS}$ & 40 \\
\hline 2 (pGL3) & Positive Control (1) & 17 & 33 \\
\hline 3 (pGL4.13) & Treatment (1) & 50 & 150 \\
\hline 3 (pGL4.13) & Negative Control (1) & $150 \mu \mathrm{L} \mathrm{PBS}$ & 40 \\
\hline 3 (pGL4.13) & Positive Control (1) & 50 & 0 \\
\hline
\end{tabular}

\section{Luciferase Assay}

Luciferase protein activity was measured using the luciferase assay reporter system (Promega, Southampton, UK). For trial 1 and 2, a mortar and pestle were used for tissue disruption, and tissue was lysed by adding 1:20 (w/v) tissue protein extraction reagent (T-PER) (ThermoFisher, Loughborough, UK) and placing samples on ice for 1 hour on a shaker. Samples were centrifuged at $10,000 \mathrm{~g}$ for 5 minutes. For trial 3, a mortar and pestle were used for tissue disruption, and tissue was homogenized in 1:20 (w/v) T-PER two times for 30 seconds at $30 \mathrm{kHz}$ with bead-milling in TissueLyser (Qiagen, Haan, Germany). Tissue was subject to three freeze thaw cycles and centrifuged at $10,000 \mathrm{~g}$ for 5 minutes. All reactions were set-up on 96 well-plates with $100 \mu \mathrm{L}$ luciferase assay reagent (Promega, Southampton, UK) and 20 $\mu \mathrm{L}$ of sample supernatant. Luciferase activity was measured in counts per second for 10 seconds using the PerkinElmir 2030 plate reader (Perkin Elmer, Singapore, Singapore).

\section{E. Protein Assay}

Total protein concentration of mouse tissue samples used in the luciferase assay was measured using the Pierce BCA protein assay (ThermoFisher, Loughborough, UK). The protein assay was performed according to the manufacturer's protocol (ThermoFisher, Loughborough, UK).

\section{F. Literature Review}

A literature review was conducted using PubMed database to identify studies between January 1990 and March 2018 with the following search terms: 'pre-eclampsia', 'preeclampsia', 'miR', and 'microRNA'. The search strategy was to identify screening studies investigating differentially expressed placental miRNAs in $3^{\text {rd }}$ trimester preeclamptic patients compared to normotensive controls. Data of miRNAs was collected based on significance defined by the study (e.g. p-value and fold change).

Significantly differentially expressed placental miRNAs detected by three or more screening studies were identified. In turn, selected miRNAs were assessed for presence of rat orthologs, studies supporting a role for the miRNA in the placenta, and validation in placenta in at least one either fully or partially independent cohort. Selected miRNAs were considered as candidates if they met all three criteria. 


\section{G. Rat Placental MicroRNAs}

Whole placental tissue was collected on gestational day 18 from four control (saline treated) and four preeclamptic (1000 $\mathrm{ng} / \mathrm{kg} / \mathrm{min}$ Ang II treated) spontaneously hypertensive stroke prone (SHRSP) rats, in which Ang II has been administered from gestational day 10.5 to induce superimposed PE [14].

\section{H. RNA Extraction}

RNA extractions were performed using miRNeasy mini kits (QIAGEN, Manchester, UK), according to the manufacturer's protocol December 2014. Rat placental tissue was homogenised in QIAzol Lysis Reagent twice for 30 seconds at $25 \mathrm{kHz}$ with Polytron PT2100 Benchtop Homogenizer with $3 \mathrm{~mm}$ dispersing aggregates (KINEMATICA, Luzern, Switzerland).

\section{MicroRNA Reverse Transcription}

Reverse transcription of rat RNA templates was performed with TaqMan ${ }^{\mathrm{TM}}$ MicroRNA Reverse Transcription Kit (ThermoFisher, Vilnus, Lithuania) according the manufacturer's protocol TaqMan ${ }^{\circledR}$ Small RNA Assays USER GUIDE, using a template specific stem loop primer supplied with the Applied Biosystems ${ }^{\mathrm{TM}} 20 \mathrm{X}$ TaqMan ${ }^{\mathrm{TM}}$ MicroRNA Assays (Table 2). All reactions were set-up in 96 well plates (Starlab, Milton Keynes, UK) and thermocycling was performed on the MJ research Tetrad PTC-225 Thermal Cycler (MJ research, Ramsey, USA).

TABLE II. TAQMAN ${ }^{\mathrm{TM}}$ MICRORNA ASSAY NAMES AND IDS

\begin{tabular}{|l|c|}
\hline \multicolumn{1}{|c|}{ Assay name } & Assay ID \\
\hline Rno-miR-363-3p & 001355 \\
\hline Hsa-miR-223 & 000526 \\
\hline Hsa-miR-210 & 000512 \\
\hline Hsa-miR-195 & 000494 \\
\hline Hsa-miR-181a & 000480 \\
\hline Hsa-miR-30a-5p & 000417 \\
\hline Hsa-miR-29b & 000413 \\
\hline Hsa-miR-16 & 000391 \\
\hline U87 & 001712 \\
\hline
\end{tabular}

\section{J. MicroRNA TaqMan $q R T-P C R$}

Amplification of rat cDNA was performed with Taqman ${ }^{\mathrm{TM}}$ Universal Master Mix II, No UNG (ThermoFisher, Carlsbad, USA), according to the manufacturer's protocol (PN 4428173B). All reactions used Applied Biosystems ${ }^{\mathrm{TM}}$ 20X TaqMan $^{\text {TM }}$ MicroRNA Assays (Table 2) in simplex. All reactions were set-up in 384 well-plates (ThermoFisher, Loughborough, UK) and thermal cycling was performed with QuantStudio 12K Flex Real-Time PCR System.

\section{K. Statistical Analysis}

Graphpad Prism 4 was used to analyse all data sets. Luciferase assay data is expressed as mean luciferase activity (counts per second (CPS) / $\mu$ g of protein) $\pm \mathrm{SD} ; \mathrm{n}=3$ (trial 1 and 3 ) and $n=4$ (trial 2) technical replicates per group. For the protein assay, linear regression was performed on standards to obtain a standard curve. Protein concentration of samples was determined from the standard curve. Taqman qRT-PCR was analysed by relative quantification (RQ) using the comparative $\mathrm{Ct}$ method; $\mathrm{n}=4$ per group. Data are shown as mean RQ (RQ min-max) \pm S.E.M and were analysed by unpaired t-test. A value of $\mathrm{p}<0.05$ was considered significant.

\section{RESULTS}

\section{A. In-vivo Trials}

In trial 1 , low levels of luciferase activity were detected in the heart of treatment mouse $1(2.56 \pm 1.95 \mathrm{CPS} / \mu \mathrm{g}$ of protein), treatment mouse $2(0.66 \pm 0.73 \mathrm{CPS} / \mu \mathrm{g}$ of protein), and treatment mouse $3(2.05 \pm 2.08 \mathrm{CPS} / \mu \mathrm{g}$ of protein), compared to background luciferase activity in the heart of the negative control mouse $(0.53 \pm 0.57 \mathrm{CPS} / \mu \mathrm{g}$ of protein) (Fig. 1A). The highest level of luciferase activity was detected in the gracilis muscle of the positive control mouse $(21.2 \pm 5.90$ $\mathrm{CPS} / \mu \mathrm{g}$ of protein) (Fig. 1B).
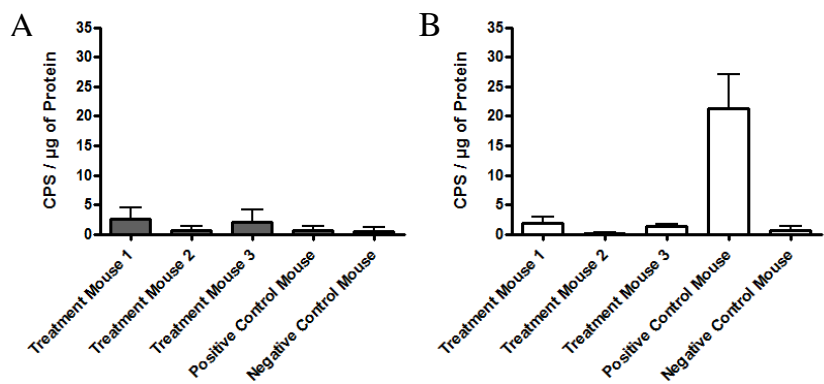

Fig. 1. Trial 1 luciferase activity in tissue from the three treatment mice, positive control mouse, and negative control mouse in A) heart and B) gracilis muscle. Data are shown as mean counts per second (CPS) / $\mu g$ of protein $\pm S D$. $n=3$ technical replicates.

In trial 2, of the heart tissue samples examined (i.e. atria and ventricles), only the atria of treatment mouse 2 (14.6 $\pm 19.5 \mathrm{CPS} / \mu \mathrm{g}$ of protein) demonstrated a signal higher than background when compared with the atria and ventricles of the negative control mouse $(7.77 \pm 2.79$ and $0.49 \pm 0.05$ $\mathrm{CPS} / \mu \mathrm{g}$ of protein, respectively) (Fig. 2A). Luciferase activity was detected in the gracilis muscle of the positive control mouse (6.17 $\pm 4.51 \mathrm{CPS} / \mu \mathrm{g}$ of protein) (Fig. 2B).
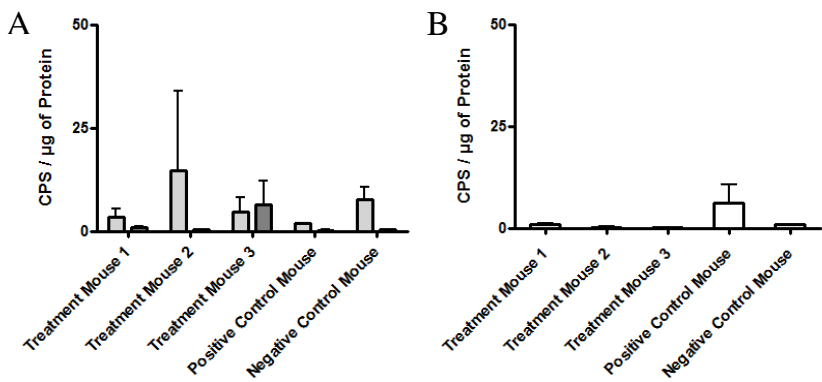

Fig. 2. Trial 2 luciferase activity in tissue from the three treatment mice, positive control mouse, and negative control mouse in A) ventricles and B) gracilis muscle. Data are shown as mean counts per second (CPS) / $\mu$ g of protein $\pm S D$. $n=4$ technical replicates.

In trial 3 , luciferase activity was detected in the ventricles and atria of the treatment mouse $(42.2 \pm 4.39$ and $2.51 \pm 0.77 \mathrm{CPS} / \mu \mathrm{g}$ of protein, respectively) (Fig. 3A), greater than background activity in the ventricles and atria of the negative control mouse $(0.29 \pm 0.38$ and $1.49 \pm 0.53 \mathrm{CPS} / \mu \mathrm{g}$ of protein, respectively) (Fig 3A). Highest luciferase activity was detected in the gracilis muscle of the positive control mouse $(1,330.3 \pm 643.8 \mathrm{CPS} / \mu \mathrm{g}$ of protein) (Fig. 3B). 

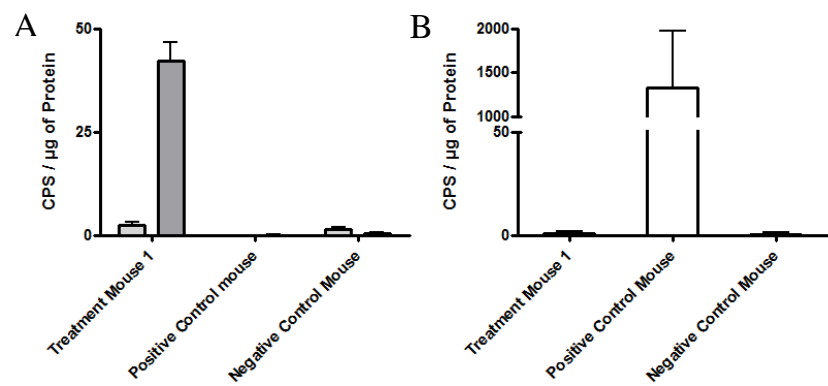

Fig. 3. Trial 3 luciferase activity in tissue from the treatment mouse, positive control mouse, and negative control mouse in A) ventricles and B) gracilis muscle. Data are shown as mean counts per second (CPS) / $\mu$ g of protein $\pm S D . n=3$ technical replicates.

\section{B. Literature Review}

14 miRNAs were detected by three or more screening studies (miR-584-5p, -542-3p, -363-3p, 301a-3p, -223-3p, 214-3p, -210-3p, -195-5p, -193b-3p, -181a-5p, -30a-5p, -29b$3 p,-16-5 p$, and $-1-3 p$ ) (data not shown). Of the 14 miRNAs, eight were selected as candidates (miR-363-3p, -223-3p, -2103p, -195-5p, -181a-5p, -30a-5p, 29b-3p, and -16-5p) based on presence of a rat ortholog, previous studies supporting a role for the miRNA in the placenta, and validation of the miRNA in the placenta in an independent cohort (data not shown).

\section{Placental MicroRNA Expression in a PE Rat Model}

There was a significant increase in the relative expression of miR-223 (1.46-fold (0.12-0.13), *p<0.05) (Fig. 4A) and significant decrease in miR-181a (0.76-fold (0.04-0.04), $* \mathrm{p}<0.05$ ) (Fig. 4B) in the placenta of the PE rat model. All other miRNAs did not reach significance (data not shown).
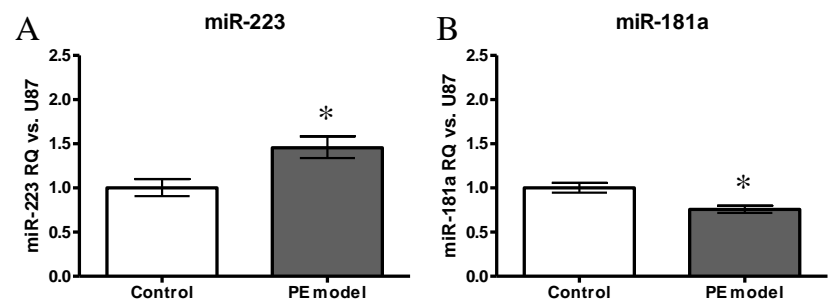

Fig. 4. Taqman qRT-PCR for evaluation of mRNA expression in the placenta of a PE rat model of candidate miRNAs A) mir-223 and B) mir181a. control, $P E$ model. Data are shown as mean RQ $\square$ S.E.M, $n=4$ per group, analysed using unpaired t-test, *p $<0.05$

\section{DISCUSSION}

The purpose of this study was to develop a protocol for US and MB gene delivery by conducting proof-of-concept trials to demonstrate site-specific gene transfection. Following adaptations to the protocol, moderate site-specific gene transfection was evident in the ventricles of a treatment mouse, notably distinguishable from background activity. This provides a basic gene delivery protocol with potential applicability to the placenta. Of eight candidate miRNAs, two miRNAs were significantly differentially expressed in PE rat model placentas. MiR-181a and miR-223 are potential targets for US and MB gene delivery to the placenta in PE.

Three previous US and MB gene delivery studies have utilized the Acuson Sequoia-512 system and 15L8 probe with SonoVue MBs and plasmid DNA injected via the tail vein to target the heart in mice, demonstrating successful gene transfection [15-17]. In trial 1, low levels of gene transfection with high variation were seen in the hearts of all treatment mice. Previous US and MB gene delivery studies targeting the heart have found gene transfection differs between regions of the heart $[18,19]$. Hence, for trial 2, hearts were dissected into the atria and ventricle, with the aim of reducing variation. In trial 2, gene transfection was detected in the atria of treatment mouse 2. Past studies have found greater transfection efficiency in the ventricles compared to the atria. One study in rats found lowest transfection in the atria compared to the ventricles when holding the probe stationary or in motion [18]. In a study in mice, where the probe was held stationary, lower gene transfection efficiency was also seen in the atria compared to the ventricles [19]. High expression in the atria in trial 2 may be attributed to probe placement. In the current study, the probe was held stationary in a parasternal long axis view, following a previous protocol [17]. This view primarily consists of the left ventricle, though the left atrium and a portion of the right ventricle are within view. Human technical variability in probe placement and biological variability in mice may have contributed to differences in the targeted region. In turn, probe placement was carefully considered in the subsequent trial.

As seen in past studies [18, 19], in trial 3, a moderate level of gene transfection was evident in the ventricles of the treatment mouse and lower levels in the atria. In comparison to trial 1 and 2 , gene transfection efficiency was notably higher in trial 3. Though previous studies have shown successful gene transfection with pGL3 in a variety of organs and species [20,21], the improved gene transfection levels in trial 3 likely reflects use of pGL4.13 in place of pGL3. pGL3 vectors contain the luciferase gene $l u c+[22]$, while pGL4 vectors harbour a synthetically engineered luciferase gene, $l u c 2$, that displays enhanced expression [23]. Finally, reduced variation and background signals likely reflect changes made to the luciferase assay in terms of tissue homogenisation.

MiR-181a and -223 may represent suitable targets for GT applications in PE, with in-vitro studies demonstrating their role in modulating properties of placental cells. MiR-181a has shown to inhibit trophoblast migration and invasion [24] and mesenchymal stem cell proliferation [25] and miR-223 has shown to promote trophoblast survival and invasion [32]. However, screening studies consistently report miR-181a is upregulated in placentas of PE patients [26-30], in contrast to the downregulation of miR-181a seen in the PE rat model. This may be attributed to the types of PE under study, supported by previous studies showing differences in miRNA expression between subtypes [27, 31]. For miR-223, screening studies in placentas of PE patients have reported both an upregulation [33-35] and downregulation [27, 28, 3639]. A study by our group is extending investigations to include miRNA candidate studies in PE patients and examine miRNA expression between rat placental layers in order to elucidate the reasons behind the contrasting findings.

Overall, this study provides a proof-of-concept protocol for potential applicability to the placenta, with results showing site-specific gene transfection in the heart of a treatment mouse. This study also identifies potential miRNAs of interest as targets for GT applications. Targeting placental miRNAs represents a novel application of US and MB gene delivery and potentially new treatment strategy for PE. 


\section{REFERENCES}

[1] Anguela, X.M. and K.A. High, Entering the Modern Era of Gene Therapy. Annu Rev Med, 2019. 70: p. 273-288.

[2] Ginn, S.L., et al., Gene therapy clinical trials worldwide to 2017: An update. J Gene Med, 2018. 20(5): p. e3015.

[3] Nayerossadat, N., T. Maedeh, and P.A. Ali, Viral and nonviral delivery systems for gene delivery. Adv Biomed Res, 2012. 1: p. 27.

[4] Rychak, J.J. and A.L. Klibanov, Nucleic acid delivery with microbubbles and ultrasound. Adv Drug Deliv Rev, 2014. 72: p. 8293.

[5] Newman, C.M. and T. Bettinger, Gene therapy progress and prospects: ultrasound for gene transfer. Gene Ther, 2007. 14(6): p. 465-75.

[6] Duley, L., The global impact of pre-eclampsia and eclampsia. Semin Perinatol, 2009. 33(3): p. 130-7.

[7] Duley, L., et al., Antiplatelet drugs for prevention of pre-eclampsia and its consequences: systematic review. BMJ, 2001. 322(7282): p. 32933.

[8] Duley, L., S. Meher, and L. Jones, Drugs for treatment of very high blood pressure during pregnancy. . Cochrane Database Syst Rev, 2013(7): p. CD001449.

[9] Abalos, E., et al., Antihypertensive drug therapy for mild to moderate hypertension during pregnancy (Review). Cochrane Database Syst Rev, 2007. 24(1): p. CD002252.

[10] Staines-Urias, E., et al., Genetic association studies in pre-eclampsia: systematic meta-analyses and field synopsis. Int J Epidemiol, 2012. 41(6): p. 1764-75.

[11] Sheikh, A.M., et al., Systematic Review of Micro-RNA Expression in Pre-Eclampsia Identifies a Number of Common Pathways Associated with the Disease. PLoS One, 2016. 11(8): p. e0160808.

[12] Chen, D.B. and W. Wang, Human placental microRNAs and preeclampsia. Biol Reprod, 2013. 88(5): p. 130.

[13] Yan, T., et al., Assessment of therapeutic efficacy of miR-126 with contrast-enhanced ultrasound in preeclampsia rats. Placenta, 2014. 35(1): p. 23-9.

[14] Morgan, H.L., et al., Modeling Superimposed Preeclampsia Using Ang II (Angiotensin II) Infusion in Pregnant Stroke-Prone Spontaneously Hypertensive Rats. Hypertension, 2018. 72(1): p. 208-218.

[15] Alter, J., et al., Microbubble stability is a major determinant of the efficiency of ultrasound and microbubble mediated in vivo gene transfer. Ultrasound Med Biol, 2009. 35(6): p. 976-84

[16] Browning, R.J., et al., Influence of needle gauge on in vivo ultrasound and microbubble-mediated gene transfection. Ultrasound Med Biol, 2011. 37(9): p. 1531-7.

[17] Browning, R.J., et al., Effect of albumin and dextrose concentration on ultrasound and microbubble mediated gene transfection in vivo. Ultrasound Med Biol, 2012. 38(6): p. 1067-77.

[18] Geis, N.A., et al., Spatial distribution of ultrasound targeted microbubble destruction increases cardiac transgene expression but not capillary permeability. Ultrasound Med Biol, 2009. 35(7): p. 1119-26.

[19] Kopechek, J.A., et al., Cardiac Gene Expression Knockdown Using Small Inhibitory RNA-Loaded Microbubbles and Ultrasound. PLoS One, 2016. 11(7): p. e0159751.

[20] Bekeredjian, R., et al., Ultrasound-targeted microbubble destruction can repeatedly direct highly specific plasmid expression to the heart. Circulation, 2003. 108(8): p. 1022-6.

[21] Song, S., et al., Explorations of high-intensity therapeutic ultrasound and microbubble-mediated gene delivery in mouse liver. Gene Ther, 2011. 18(10): p. 1006-14.
[22] PromegaCorporation, pGL3 Luciferase Reporter Vectors, in Instructions For Use of Products, P. Corporation, Editor. 2015: Madison, USA.

[23] PromegaCorporation, pGL4 Luciferase Reporter Vectors, in Instructions for Use of Products, P. Corporation, Editor. 2015: Madison, USA.

[24] Wu, L., et al., miR-181a-5p suppresses invasion and migration of HTR8/SVneo cells by directly targeting IGF2BP2. Cell Death Dis, 2018. 9(2): p. 16.

[25] Liu, L., et al., MicroRNA-181a regulates local immune balance by inhibiting proliferation and immunosuppressive properties of mesenchymal stem cells. Stem Cells, 2012. 30(8): p. 1756-70.

[26] $\mathrm{Hu}$, Y., et al., Differential expression of microRNAs in the placentae of Chinese patients with severe pre-eclampsia. Clin Chem Lab Med, 2009. 47(8): p. 923-9.

[27] Zhu, X.M., et al., Differential expression profile of microRNAs in human placentas from preeclamptic pregnancies vs normal pregnancies. Am J Obstet Gynecol, 2009. 200(6): p. 661.e1-7.

[28] Xu, P., et al., Variations of microRNAs in human placentas and plasma from preeclamptic pregnancy. Hypertension, 2014. 63(6): p. 1276-84.

[29] Jiang, F., et al., Upregulation of microRNA335 and microRNA584 contributes to the pathogenesis of severe preeclampsia through downregulation of endothelial nitric oxide synthase. Mol Med Rep, 2015. 12(4): p. 5383-90

[30] Zhang, C., et al., Placental miR-106a 363 cluster is dysregulated in preeclamptic placenta. Placenta, 2015. 36(2): p. 250-2.

[31] Yang, S., et al., Deregulated microRNA species in the plasma and placenta of patients with preeclampsia. Mol Med Rep, 2015. 12(1): p. 527-34.

[32] Meng, H., et al., MiR-223 promotes trophoblast cell survival and invasion by targeting STAT3 in preeclampsia. Int J Clin Exp Med 2017. 10(4): p. 6577-6585.

[33] Guo, L., et al., A comprehensive survey of miRNA repertoire and 3' addition events in the placentas of patients with pre-eclampsia from high-throughput sequencing. PLoS One, 2011. 6(6): p. e21072.

[34] Mayor-Lynn, K., et al., Expression profile of microRNAs and mRNAs in human placentas from pregnancies complicated by preeclampsia and preterm labor. Reprod Sci, 2011. 18(1): p. 46-56.

[35] Betoni, J.S., et al., MicroRNA analysis in placentas from patients with preeclampsia: comparison of new and published results. Hypertens Pregnancy, 2013. 32(4): p. 321-39.

[36] Ishibashi, O., et al., Hydroxysteroid (17-beta) dehydrogenase 1 is dysregulated by miR-210 and miR-518c that are aberrantly expressed in preeclamptic placentas: a novel marker for predicting preeclampsia. Hypertension, 2012. 59(2): p. 265-73.

[37] Choi, S.Y., et al., MicroRNA expression profiles in placenta with severe preeclampsia using a PNA-based microarray. Placenta, 2013. 34(9): p. 799-804.

[38] Zhao, G., et al., Differential expression of microRNAs in deciduaderived mesenchymal stem cells from patients with pre-eclampsia. J Biomed Sci, 2014. 21: p. 81 .

[39] Vashukova, E.S., et al., Placental microRNA expression in pregnancies complicated by superimposed preeclampsia on chronic hypertension. Mol Med Rep, 2016. 14(1): p. 22-32.

[40] Zhang, C., et al., Placental miR-106a 363 cluster is dysregulated in preeclamptic placenta. Placenta, 2015. 2(36): p. 250-2. 